\title{
Overview and Status of the Laser Communication Relay Demonstration
}

\author{
E. Luzhansky, B. Edwards, D. Israel, D. Cornwell*, J. Staren, N. Cummings, T. Roberts ${ }^{\star \star}$, R. Patschke \\ NASA, Goddard Space Flight Center, Greenbelt, MD, *NASA, Headquarters, Washington, DC, ** Jet \\ Propulsion Laboratory, Pasadena, CA
}

\begin{abstract}
NASA is presently developing first all optical high data rate satellite relay system, LCRD. To be flown on commercial geosynchronous satellite, it will communicate at DPSK and PPM modulation formats up to 1.244 Gbps. LCRD flight payload is being developed by NASA's Goddard Space Flight Center. The two ground stations, one on Table Mountain in CA, developed by NASA's Jet Propulsion Laboratory and another on Hawaiian island will enable bi-directional relay operation and ground sites diversity experiments.

In this paper we will report on the current state of LCRD system development, planned operational scenarios and expected system performance.
\end{abstract}

\section{INTRODUCTION}

The communications link between space-borne observatories and the Earth has long been a critical mission systems design constraint. The information from a scientific or exploration discovery has to be transmitted to Earth; the more data sent back, the better the probability that the mission will produce more valuable science. Several technologies such as higher data bandwidth radio frequency (RF) communications and lossless data compression have improved the capability over time, but are failing to keep pace with the needs of advanced instrumentation that can be flown in space today. NASA is developing a free-space optical (FSO) communications technologies ${ }^{1,2.3}$ in order to remove data throughput bottleneck. For the same mass and power, an FSO system can provide higher data rates than a comparable RF system. Besides higher data throughput, FSO systems reduce user burden in terms of required size, weight, and power (SWaP) for transmit and receive terminals.

The Laser Communications Relay Demonstration (LCRD) mission provides a space-based technology demonstration of optical communications, leveraging work done on the previous NASA missions, including Lunar Laser Communication Demonstration (LLCD) ${ }^{4}$. The mission is scheduled to launch in mid-2019. LCRD is a joint project between NASA Goddard Space Flight 
Center (GSFC), NASA Jet Propulsion Laboratory (JPL), and MIT Lincoln Laboratory. The project office resides at GSFC.

LCRD provides two years of high data rate optical communications in an operational environment, demonstrating that optical communications can meet NASA's growing need for higher data rates while also enabling lower power, lower mass communications systems on spacecraft. In addition, LCRD's architecture enables it to serve as a developmental testbed for additional methods, including symbol coding, ranging, link layer protocols, and network layer protocols. The dual optical link system also enables LCRD to become the first step in demonstrating optical communications for the Next Generation Tracking and Data Relay Satellite. The LCRD mission advances optical communications technology toward infusion into operational systems, while growing the capabilities of industry sources to produce affordable optical communications systems and components.

\section{LCRD CONCEPT OF OPERATIONS}

3.

\section{Main operation}

The LCRD mission objectives are to demonstrate bidirectional optical communications between geosynchronous Earth orbit (GEO) and Earth, measure and characterize the system performance over a variety of conditions and provide an on orbit capability for test and demonstration of standards for optical relay communications ${ }^{5}$.

LCRD will consist of a payload with two space terminals hosted on a geostationary Earth orbit (GEO) platform, an operations control center, and optical ground stations. The design reference mission for LCRD is illustrated in Figure 1. LCRD will employ simulators to demonstrate forward and return relay links and direct uplink/downlink. The flexibility and scalability of the LCRD architecture enables support to terrestrial, air-borne, and Low Earth Orbit (LEO) users. LCRD will also demonstrate optical communications networking capabilities including the use of an LCRD Mission Operations Center (LMOC), multiple Optical Ground Stations (OGSs), OGS handovers, degraded operations, user service recovery from link interruption due to clouds, operating through orbital events and spacecraft maneuvers, and coordinated network flight and ground segment operations. 


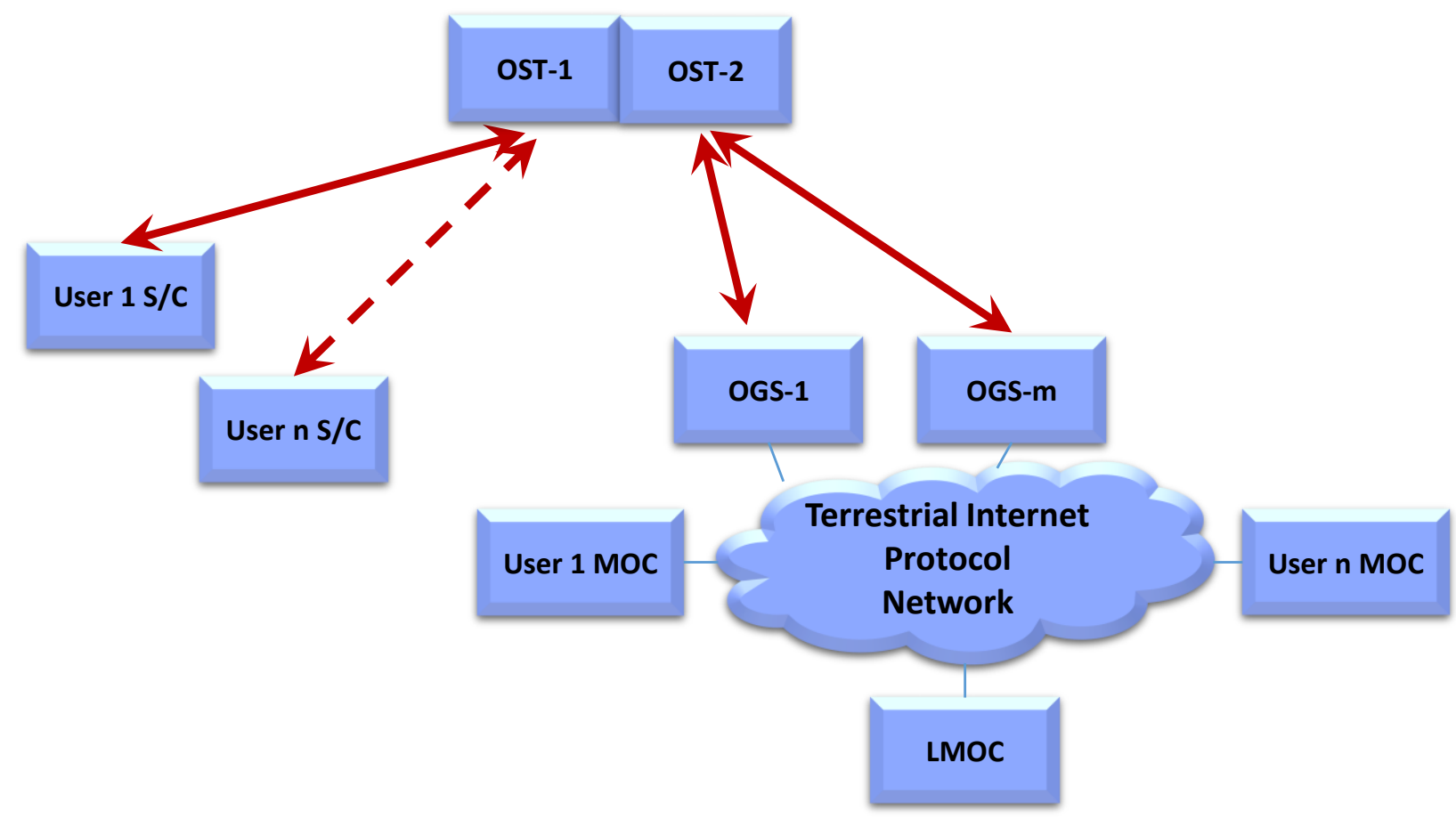

Figure 1 LCRD Design Reference Mission

Experiments with LCRD

LCRD will have a dedicated Experiment Operations (ExpO) team focused on developing mission experiments and analyzing the results. This team will be comprised of the mission Principal Investigator, Co-Investigators, and other experts in optical communications and communications networks. Functions of the ExpO team include experiments designs, execution and postexperiment analysis, and conduction of anomaly investigations, analysis, and resolution.

\section{SPACE PAYLOAD}

The Flight (or space) payload is designed to support operations for a minimum of two years in geostationary orbit environment with a mission goal of five years.

The location of the GEO satellite will be within $107 \mathrm{~W}$ to $172 \mathrm{~W}$ range (Figure 2). This range was chosen to insure minimum elevation of 20 degrees above horizon for LCRD ground stations. 


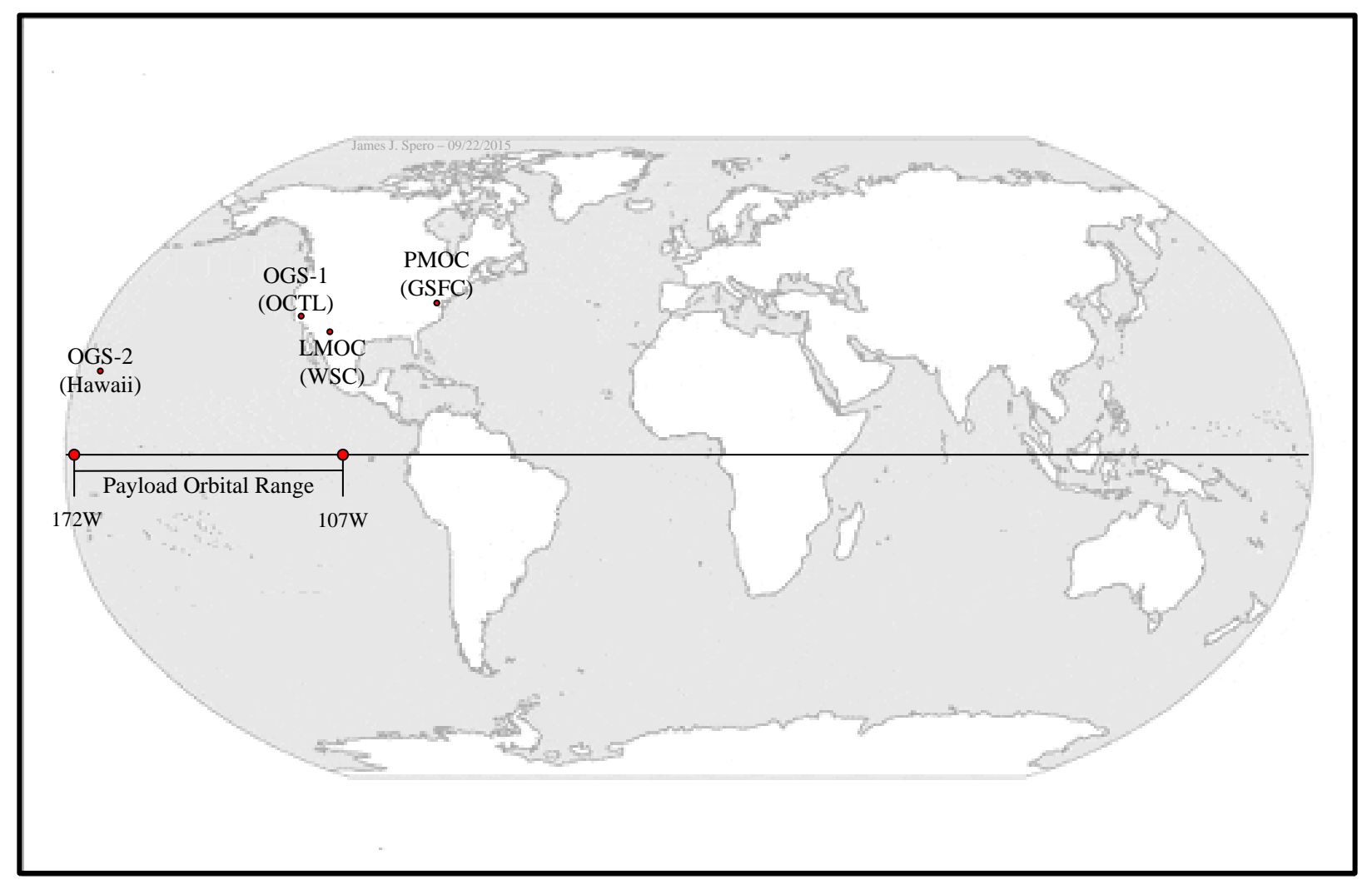

Figure 2 LCRD Operational Asset Map

The LCRD Payload includes two independent Optical Space Terminals (OSTs), which communicate via optical links with LCRD optical ground stations or flying platforms; and Space Switching Unit (SSU) which manages LCRD payload operations and routes data, commands and telemetry. Each OST includes a Controller Electronics (CE), Modem, and Optical Module (OM) subsystems. The Payload element subsystems are shown in Figure 3. 


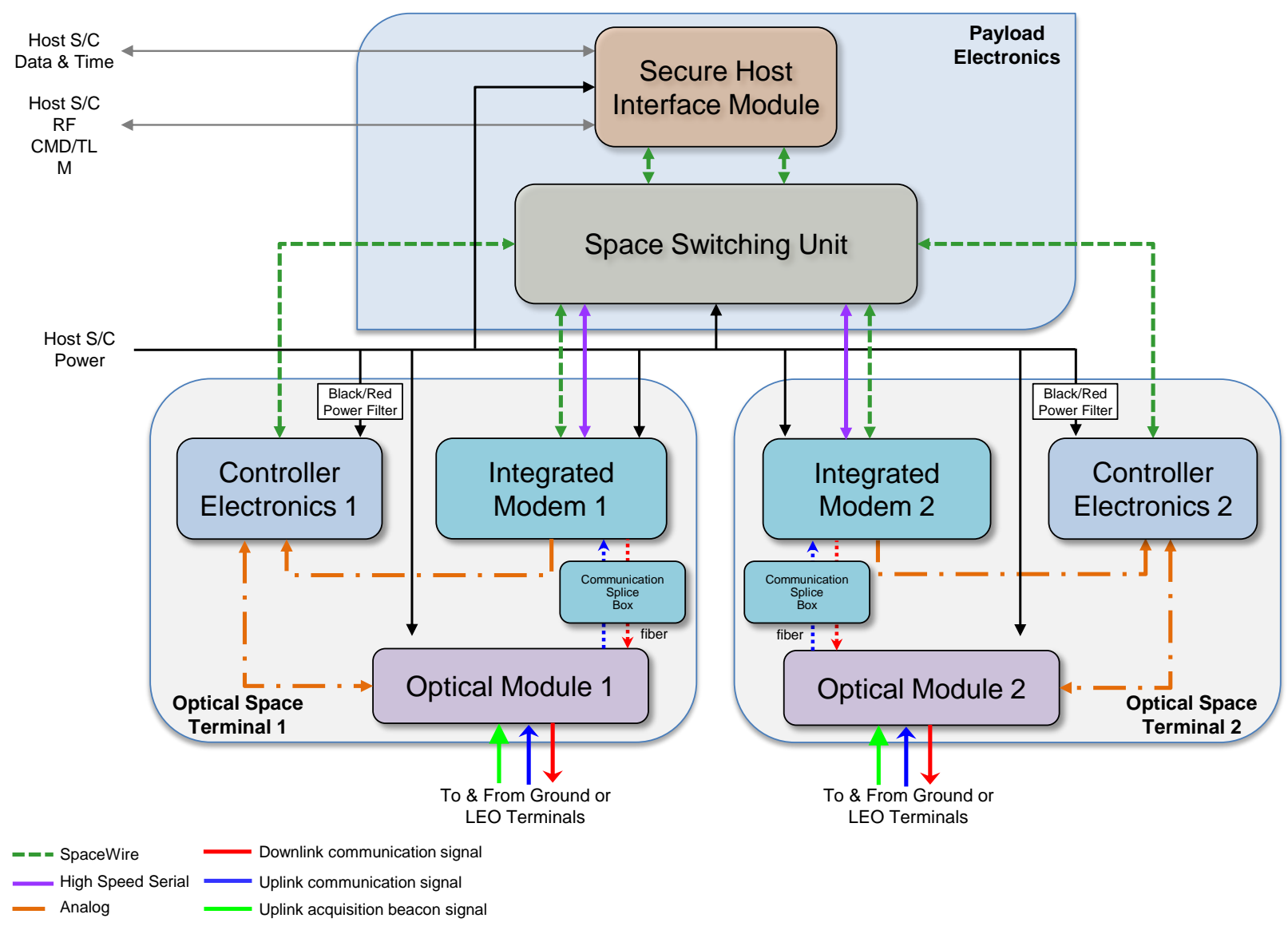

Figure 3 Payload Element Subsystems

\section{Optical Modules}

The LCRD Optical Module (OM) in each optical space terminal is a 10-cm Cassegrain telescope on a two-axis gimbal. The OM contains a Latch Cover that constrains the gimbals during launch which is released post-launch to allow gimbal motion. The OM Latch Cover incorporates a retro reflector used for Payload built in test functions and calibrations. The telescope is inertially stabilized using a magneto-hydrodynamic inertial reference unit (MIRU) that rejects highfrequency disturbances from the spacecraft interface. Transmit and receive signals are coupled to and from the telescope via single-mode optical fibers. 


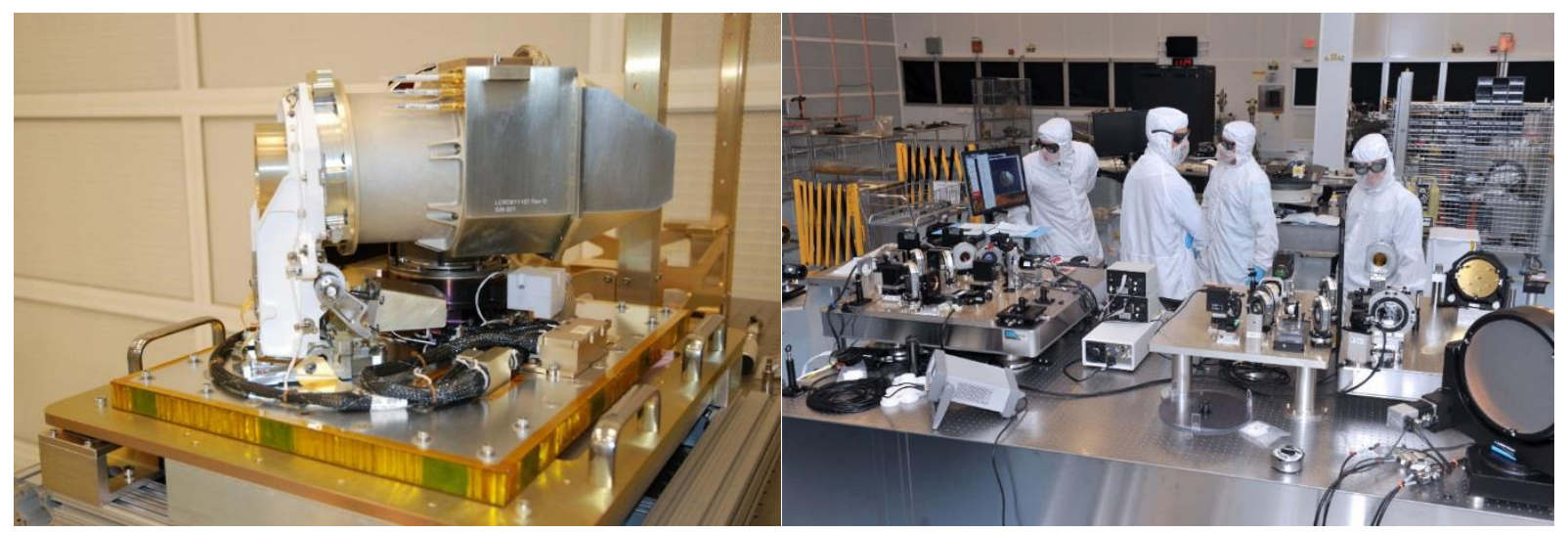

Figure 4 LCRD Optical Module and Optical Test System

All parts of Optical Module assembly will be delivered by the spring of 2016 with full OM integration planned by the end of 2016. A complex Optical Test Assembly (OTS) was built for the comprehensive OM testing prior integration into the Flight Payload

\section{Controller Electronics}

The Controller Electronics (CE) modules contain the Payload optical Pointing, Acquisition, and Tracking (PAT) software, sample feedback signals from the OM, generate control signals for pointing, acquisition and tracking (PAT) software, provide thermal control to OM telescope, and support boresight calibrations and other BIT activities. These subsystems take in attitude information from the Host spacecraft and pointing information from the LCRD ground segment. Using this information, the CEs control the pointing of the OMs.

Both Control Electronics modules are delivered and ready for payload integration.

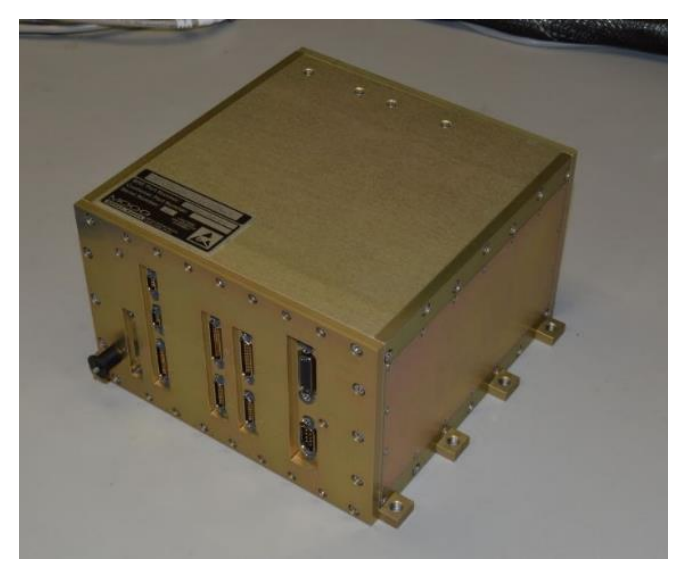

Figure 5 Control Electronics Module

\section{Space Switching Unit}

The Space Switching Unit (SSU) is the central controller for the Payload. The SSU will receive and route User data, receive and process Payload commands, and accumulate and transmit Payload telemetry.

User service data will be received from one of the communication space terminals and be routed to up to two destination ports within the SSU. 
Command and control data will be received via optical link (imbedded) or from the Host interface. The SSU will accumulate telemetry from all Payload subsystems and continuously transmit it via either path.

SSU Engineering unit is here and being tested. The flight unit here by the end of 2016

\section{Integrated modem}

Each integrated modem supports both Pulse Position Modulation (PPM) and Differential Phase

Shift Key (DPSK) waveforms. LCRD project is currently investigating On-Off Keying (OOK) modulation as possible third modulation format. OOK will be used during LCRD experiments phase of the program. LCRD modems modulation rate is 2.88 Gbps. The data rates down to $2 \mathrm{Mbps}$ will be reached via a "burst mode" format, with data bursts interspersed with "dead times" where no signal is transmitted.

Modems will be capable of providing all modulation capabilities on both the uplink and downlink, as well as with different modulation formats on each of the links to a single modem. For example, the uplink can use DPSK and the downlink can use PPM with the same hardware. The modems also include multiple BuiltIn-Test (BIT) functions enabling simple calibrations and internal modem or Payload loopback testing with the OMs. The modems will also generate test pattern data frames to be used in Direct Downlink return service demonstrations. This data can be sent alone or multiplexed with other data frames downlinked through the same modem and optical terminal.

As of January of 2016, all flight modems boards had been built. Both flight modems integration is planned for the early 2017.

In the meantime, two ground modems, functionally identical to the flight modems, were fully built and successfully tested by NASA GSFC engineers. Their performance fully meets LCRD requirements (see Figure 7 )

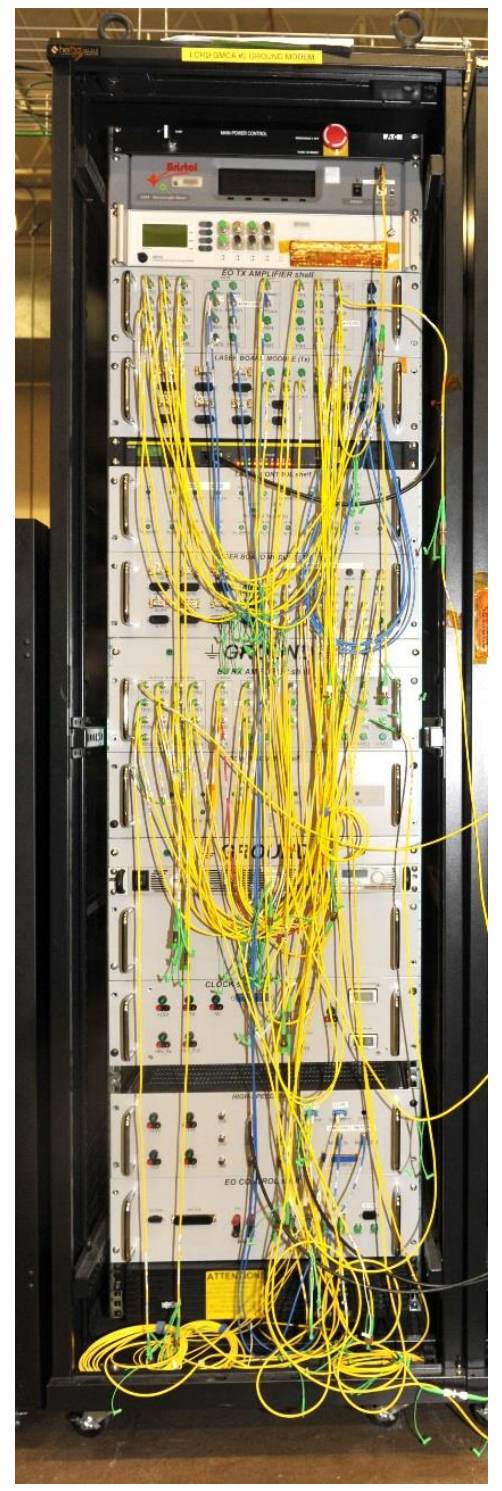

Figure 6 LCRD Ground Modem 


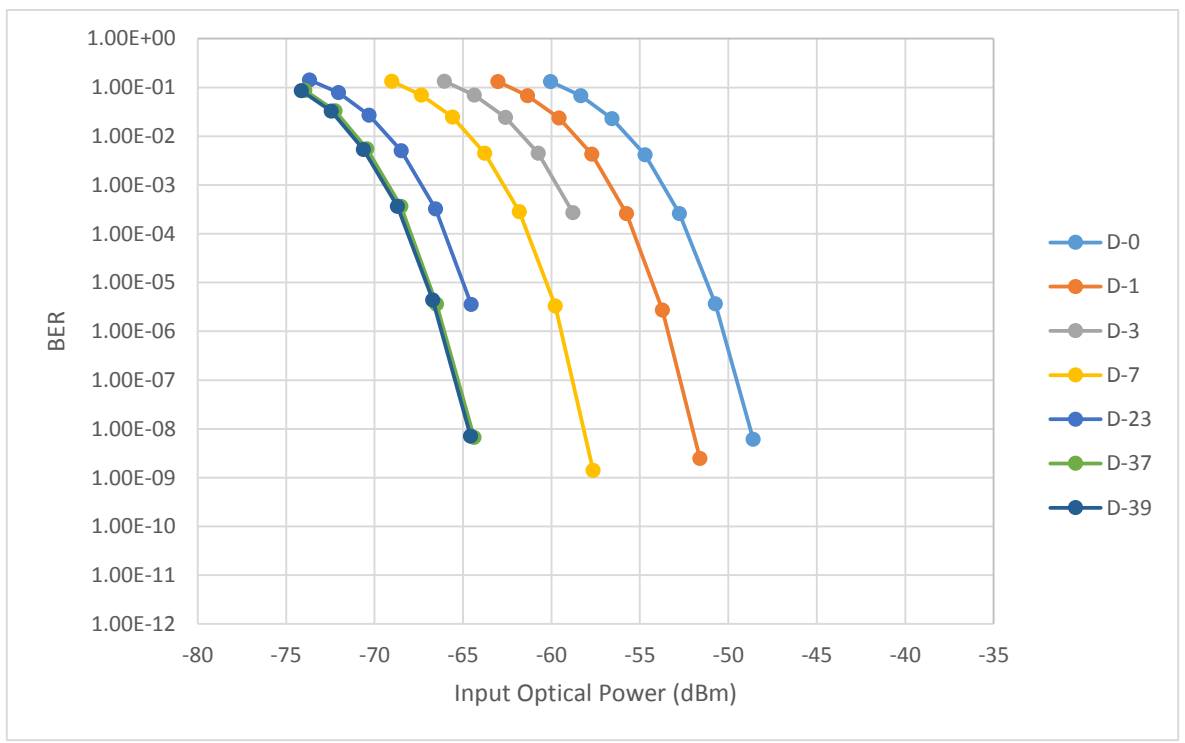

Figure 7 Ground Modem DPSK performance

\section{GROUND SEGMENT STATUS}

The LCRD ground segment consists of the LCRD Mission Operations Center (LMOC) and multiple OGSs. The primary LCRD ground station is located at the JPL OCTL facility in California. A second ground station is located in Hawaii. Both ground stations will be managed under the Optical Ground Station - eXpansion (OGS-X) Project of the Space Communications and Networking (SCaN) Program.

The LCRD ground segment will operate 24 hours per day, 7 days per week.

\section{LCRD Mission Operations Center}

The LCRD Mission Operations Center (LMOC) will be located at the White Sands Complex in New Mexico and will serve a dual role as the operations center for the LCRD Payload and the network operations center for the LCRD optical network.

As a payload operations center, the LMOC will telemeter the LCRD Flight payload to monitor its health and performance as well as commanding payload functions either via an in-band optical or through host spacecraft's RF link.

The LMOC acts as the central point of coordination for controlling the optical network and providing high-level situational awareness for the network operator. It will determine the status of optical links and communications services on the network and coordinate changes in optical network topology. 


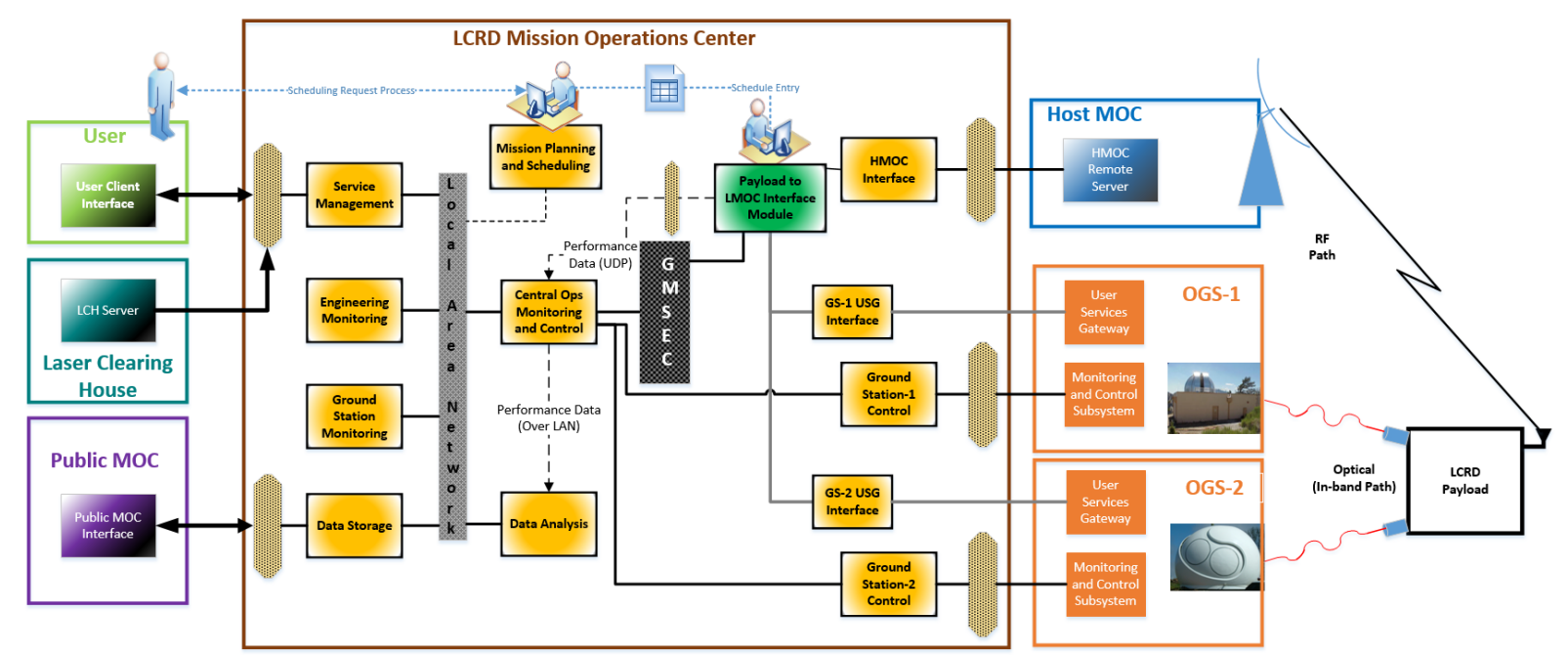

Figure 8 LCRD Mission Operations Concept

\section{Optical Ground Station}

The primary ground terminal for LCRD, Optical Ground Station 1 (OGS1) is being developed by the Jet Propulsion Laboratory (JPL) at the Optical Communications Telescope Laboratory (OCTL) telescope near Wrightwood, California. A block diagram showing interfaces between OGS1 subsystems is shown in Figure 9. 


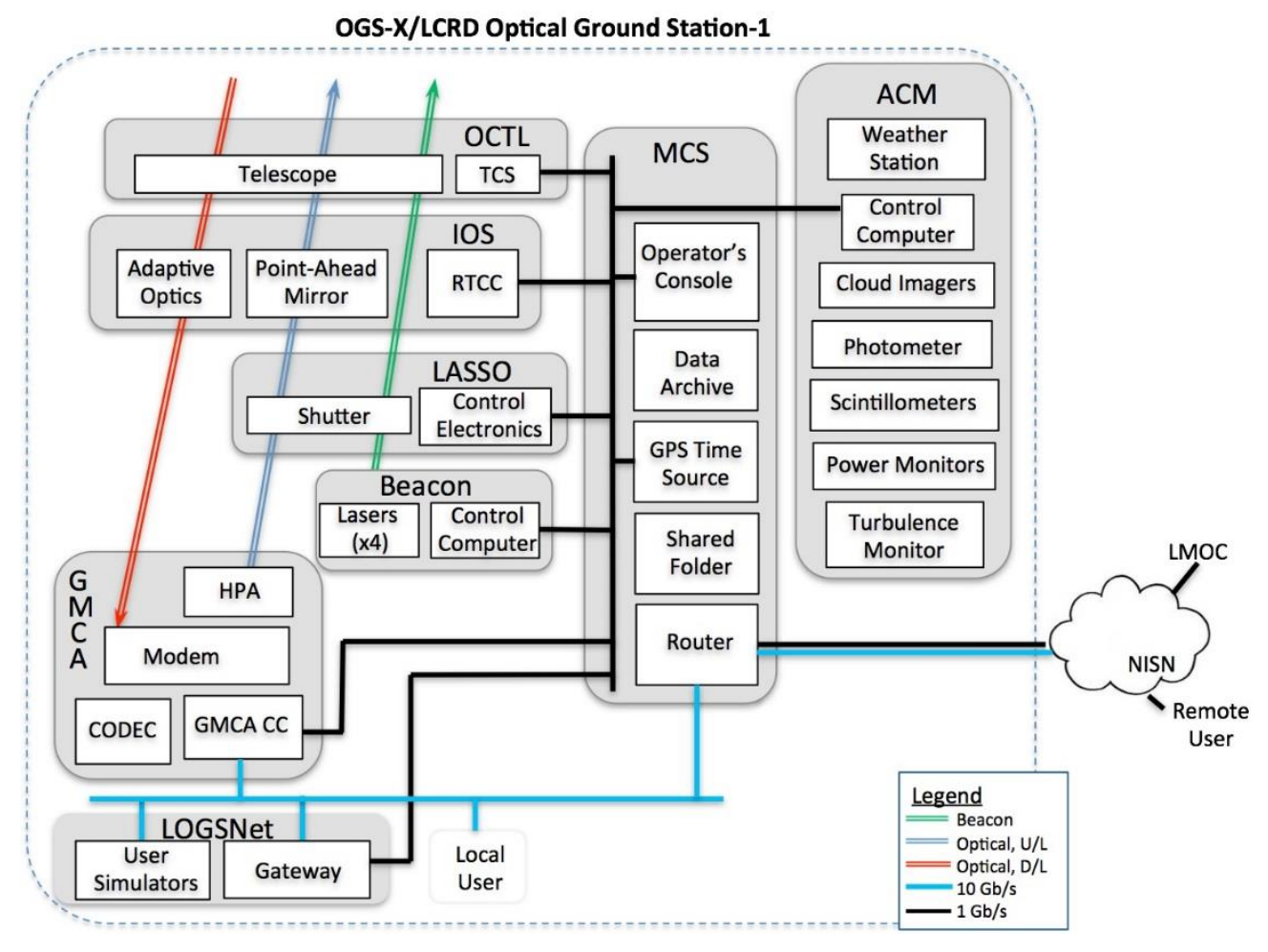

Figure 9 OGS1 block diagram

Two unique development areas of the project are the Integrated Optical System (IOS), and an extensive networking service provision, LOGSNet, described below.

Integrated Optical System: The IOS is a state-of-the-art optical system that couples the Ground Modem, Codec and Amplifier (GMCA) to the 1-meter F/76 telescope. The system contains both the transmit and beacon beam forming optics for transmitting light to the spacecraft through the 1-meter telescope, as well as a state-of-the-art Adaptive Optics (AO) system for efficiently coupling light received from the spacecraft into a single-mode-fiber-coupled detector of the GMCA.

The IOS laser beam-forming optics modify the beam of the $10 \mathrm{~W}$ modulated communication laser from the GMCA to generate a $20 \mathrm{urad}\left(1 / \mathrm{e}^{2}\right.$ diameter) beam on the sky. Because the telescope tracks the observed downlink spot from the geo-stationary payload, this optical system must also introduce an 18 urad point-ahead offset, to precisely target the position the spacecraft will be when the light pulses arrive. The IOS transmit system also takes the slowly modulated output of the four $2 \mathrm{~W}$ beacon lasers, converting them to provide 280 urad beams, all overlapping in the far field. These beams project from different portions of the telescope aperture, separated by at least $20 \mathrm{~cm}$, effectively breaking the coherence among the beams and reducing the overall beam wander and interference in the far field. The beacon beams are designed to generate a 
pattern on the sky more than 15 times wider than the point-ahead angle, so these beams are not steered to account for the light time-of flight. The IOS beacon beam forming system is similar to that used at the OCTL for the LLCD experiment in 2013-2014 ${ }^{6}$.

The bulk of the IOS consists of a state-of-the-art AO system to correct the atmosphericallyaberrated wavefronts collected by the 1-meter telescope into near-spherical wavefronts for coupling to the single-mode fiber into the GMCA. Though the OCTL telescope site has relatively good cloud-free conditions, it is frequently subject to poor seeing. The AO system is designed to be robust to pessimistic seeing conditions brought on by two factors: (1) the need for communications systems to work over a wide range of conditions, including poor seeing conditions; and (2) a lack of knowledge of the spacecraft location during the design and development of the AO system, leading to a design which must still work at elevation angles as low as 20 degrees. As a result, the AO system is designed to work in poor seeing down to $\mathrm{r} 0$ (Fried's parameter) $=2.5 \mathrm{~cm}$, and Greenwood frequencies exceeding $10 \mathrm{kHz}$. This is a significant step up in performance from the PALM 3000 AO system at the 200-inch Hale Telescope, from which the design of this AO system draws ${ }^{7}$.

The AO system splits off approximately $20 \%$ of the collected signal from the spacecraft, using that light to measure signal phase in the telescope pupil. It then corrects those phase errors using a woofer-tweeter approach, in which two deformable mirrors (DMs) are used in opticallyconjugate locations, to correct pupil seeing. A High-Order DM (HODM), with an actuator spacing corresponding to about $3.5 \mathrm{~cm}$ in the telescope pupil, is used to correct high-spatialfrequency aberrations. However, the actuators on this DM have insufficient stroke to correct for the expected high-amplitude aberrations generated by the worst seeing conditions. To correct for those, the partially-corrected wavefront from the HODM is re-imaged into a Low Order DM (LODM), which has sufficient stroke to correct the remaining high-amplitude aberrations. These systems must work rapidly in concert, using a DSP-based controller to apportion the correction between the two DMs at speeds up to $20 \mathrm{kHz}$. An additional $10 \%$ of the collected signal is directed to a scoring camera for evaluating the performance of the AO system. Model-based predictions of the fiber coupling performance indicate an expected average coupling efficiency of 0.53 in the worst (90th percentile) seeing conditions, to an average of 0.64 under nominal (50th percentile) conditions.

LOGSNet: One of the principle objectives of the LCRD mission is to demonstrate space-based networking services on a Tracking and Data Relay Satellite (TDRS)-type system. In support of this, OGS1 is developing the infrastructure to host multiple simultaneous user services through a resident User Services Gateway (USG). The USG will support up to 3 channels of coded and 3 channels of uncoded data transfer at rates prescribed by the LMOC-distributed traffic profile. Types of service being developed include (1) Symbolstream, in which user data is transferred directly as supplied bits, without additional, (2) Bitstream, in which the user data has Forward Error Correction (FEC) and data interleaving applied, (3) Advanced Orbiting Systems (AOS) protocol, developed for optical communication efficiency of space-related links, and (4) Internet 
Protocol (IP) based on an IP over CCDSD standard, the highest layer service offered by the system. This service embeds variable-length internet protocol packets into fixed-length AOS frames, for more transmission flexibility, while still incorporating the optical transmission robustness of AOS service.

The LOGSNet system also is developing simulators for fully exercising the data links, during those periods in which user data is not being transmitted. The first of these simulators is a User MOC Simulator (UMS), which simulates the setup and transfer of different types of user data at varying speeds and varying file lengths. Multiple UMS simulations can be run simultaneously based on the LMOC schedule and traffic profiles, simulating multiple users with different data types trying to establish, operate, and terminate their data links through the OGS1 USG. The UMS interfaces to the GMCA through the USG, just as a user accessing OGS1 from outside would.

A User Platform Simulator (UPS) is also being developed, to simulate the operation of an orbiting platform on the other end of the space-based relay link. Multiple platforms may simultaneously operate, again with varying data types, according to the schedules and traffic profiles prescribed by the LMOC. A principle difference between the UPS and the UMS is that the UPS accesses the GMCA directly, rather than going through the USG.

The final LOGSNet simulator is the Channel Simulator (ChannelSim), which introduces dropouts, and interruptions to the simulated data, similar to the types of perturbations expected from communicating to other assets through real free-space channels. This unit simulates the effects on the service data associated with spacecraft pointing jitter and atmospheric-turbulenceinduced dropouts.

More detailed information about these and other subsystems of OGS1 is to be found in the OGS1 companion paper $^{8}$.

\section{LCRD PERFORMANCE}

LCRD relay system is expected to perform with sufficient margin to insure reliable operation over varying channel condition. Figure 10 demonstrates expected performance on data relay between two ground stations at nominal atmospheric conditions. Solid lines represent ideal LCRD coding capability for the nominal channel. Dots represent expected performance for four cases where data originating and terminating points can be either at Ground Station 1 (GS1) or Ground Station 2 (GS2). 


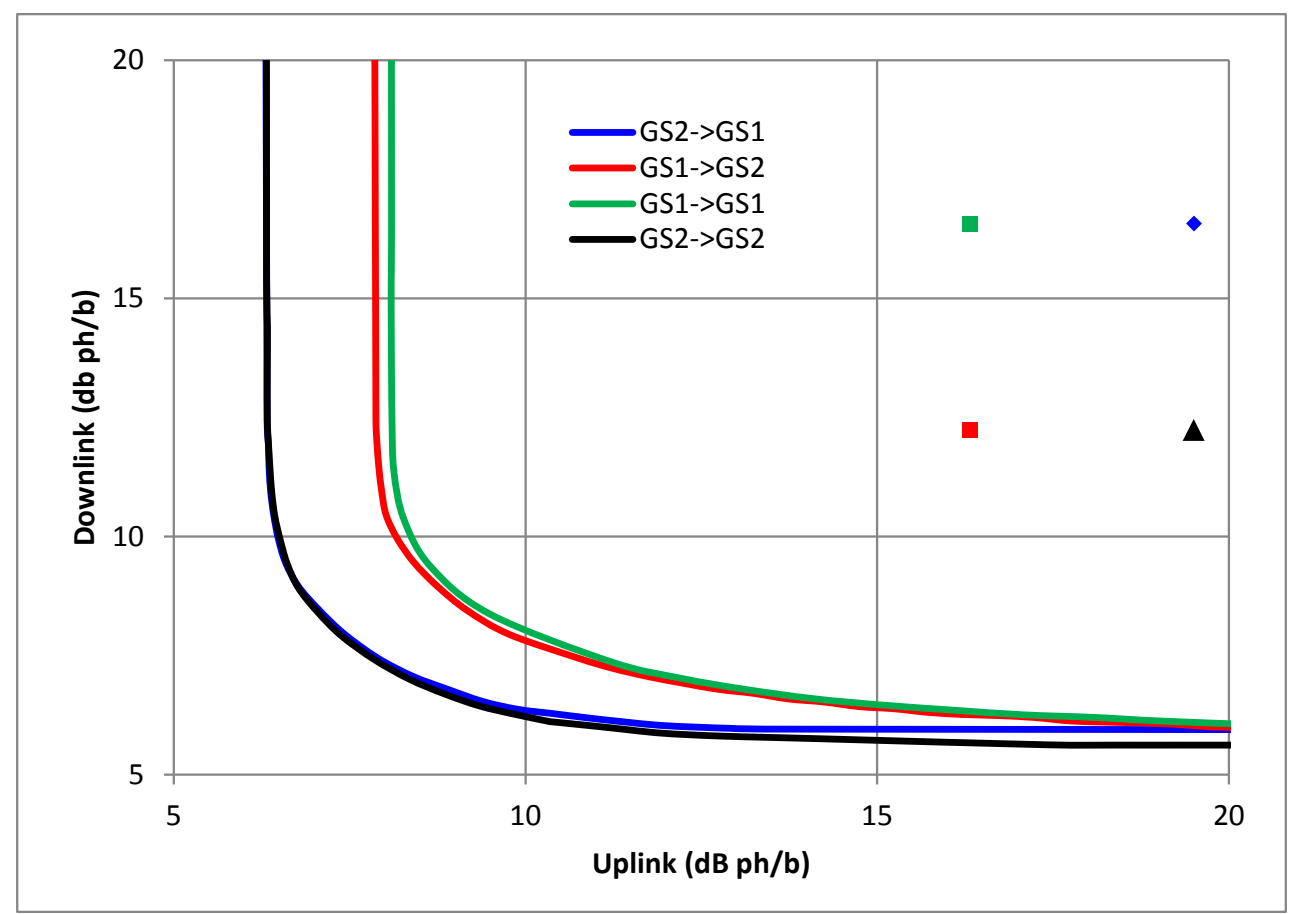

Figure 10 Notional DPSK Coding Curves and Operating Points

\section{SUMMARY}

The Laser Communication Relay Demonstration, first all optical relay communication system, is currently under the development by the team of NASA's Goddard Space Flight Center, NASA's Jet Propulsion Laboratory and MIT Lincoln Laboratory engineers and scientist. All elements of Space and Ground segments are either complete or at the final stages of integration. LCRD ground modems successfully met required bit error rate performance. The LCRD development is on schedule for the planned launch in 2019.

\section{REFERENCES}

[1] Donald M. Cornwell, "NASA's optical communications program for 2015 and beyond”, Proc. SPIE 9354, Free-Space Laser Communication and Atmospheric Propagation XXVII, 93540E (March 16, 2015)

[2] Bernard L. Edwards, David J. Israel, "Commercialization and Standardization Progress towards an Optical Communications Earth Relay”, 2015 IEEE Aerospace Conference 
[3] Bernard L. Edwards, “An Overview of NASA's Latest Efforts in Optical Communications", International Conference on Space Optical Systems (ICSOS) October 2015

[4] D. M. Boroson ; B. S. Robinson ; D. A. Burianek ; D. V. Murphy ; A. Biswas, "Overview and status of the Lunar Laser Communications Demonstration", Proc. SPIE 8246, Free-Space Laser Communication Technologies XXIV, 82460C (February 9, 2012)

[5] Bernard L. Edwards and Andrew Fletcher, "NASA's Laser Communications Relay Demonstration Project”, Applications of Lasers for Sensing and Free Space Communications 2013, Paris France, 27 October-1 November 2013

[6] Robinson, B.S., Boroson, D.M., Burianek, D.A., Murphy, D.V., "The lunar laser communications demonstration," Proceedings, 2011 International Conference on Space Optical Systems and Applications, pp. 54-57, May 2011

[7] T. N. Truong, A. H. Bouchez, R. S. Burruss, R. G. Dekany, S. R. Guiwits, J. E. Roberts, J. C. Shelton, \&M.Troy, "Design and implementation of the PALM-3000 real-time control system," Proc. SPIE, 8447, 844702F (2012).

[8] Roberts, W. T., Ansos, D., Croonquist, A., Piazzolla, S., Roberts, L. C. Jr., Garkanian, V., Trinh, T., Wright, M. W., Rogalin, R., Wu, J., and Clare., L., "Overview of Optical Ground Station 1 of the NASA Space Communications and Navigation Program”, Proc. SPIE, 9739 (this issue). 